\title{
Comment
}

Neuroepidemiology 2015;45:255-256

DOI: $10.1159 / 000441315$

\section{Insights on the Early-Life Origins of Alzheimer's Disease: Relevance for Primary Prevention?}

Steven M. Albert

Department of Behavioral and Community Health Sciences Pitt Public Health University of Pittsburgh, Pittsburgh, Pa., USA

Most research involving the prevention of Alzheimer's disease (AD) focuses on secondary prevention, that is, on identifying people at risk of the disease and providing therapy designed to slow cognitive decline and disease progression. For example, the AntiAmyloid Treatment in Asymptomatic Alzheimer's (A4) study seeks to enroll people aged 65-85 years without current cognitive impairment but with elevated levels of brain amyloid plaque. Elevated amyloid accumulation (evident in about one-third of adults in this age range) is a risk factor for $\mathrm{AD}$ and can be identified with positron emission tomography amyloid imaging. This large randomized controlled study will test the effects of an investigational anti-amyloid antibody on rates of cognitive decline over 3 years as well as changes in biomarkers of disease progression, such as brain volume, cortical thinning and hippocampal atrophy [1]. Using positron emission tomography imaging to identify early, asymptomatic $\mathrm{AD}$ is an important strength of this trial.

Other attempts at prevention have examined midlife risk factors, mainly cardiometabolic conditions (diabetes and high glucose, obesity and metabolic syndrome, hypertension, high cholesterol) and also lifestyle factors (smoking, alcohol, diet, physical activity and stress) and disease conditions that may influence hypothesized AD pathways (depression, poor sleep and inflammation). Epidemiologic studies examining these factors and their association with risk of dementia in later life have been equivocal, and researchers who want to conduct long-term randomized controlled trials to assess the effects of midlife risk reduction on risk of $\mathrm{AD}$ (such as statins to lower cholesterol or aggressive control of hypertension across the lifespan) face many challenges.

Still, the accumulating evidence of the long latency of AD suggests that cognitive and brain changes that presage $\mathrm{AD}$ may begin much earlier in life. Identifying these early-life risk factors may suggest new prevention targets. Thus, the review of the 'early-life epidemiology' of AD by Seifan et al. [2] is welcome. If the factors identified in this review are truly relevant to risk of $\mathrm{AD}$, it may be possible to expand candidates for secondary prevention and even think about 'lifespan primary prevention', that is, interventions designed for entire populations offered early in life.

In a careful retrieval and review of nearly 35 years of research, Seifan et al. [2] identified 43 papers that examined early-life factors associated with risk of $\mathrm{AD}$. The authors limited this review to diagnosed late-onset $\mathrm{AD}$ or episodic memory difficulty or decline. They identify 3 broad mechanistic models for early-life risk factors.

In the trajectory model, illustrated by childhood socioeconomic status or genetic factors, an initial factor determines later development, with gradual accumulation of $\mathrm{AD}$ risk. For example, $A P O E 4$ is associated with reduction in cortical volume and connectivity in brain networks affected by $\mathrm{AD}$ but may also impair cognition throughout the lifespan. Children with the SORL1 variant, associated with late-onset $\mathrm{AD}$, already show white matter microstructural abnormalities. Likewise, the 1938 Aberdeen birth cohort study suggests that childhood socioeconomic status is correlated not just with school performance and measured child intelligence but also with white matter hyperintensities in late life [3]. A recent study suggests that income and brain surface area assessed by brain morphometry are associated logarithmically [4] . These findings suggest long-term risk of $\mathrm{AD}$ due to factors already present at birth or at young age.

In the latent model, an initial predisposing factor is unmasked by a later factor. As illustrated by the case of learning disabilities, early changes in a specific anatomical region predispose to $\mathrm{AD}$ pathology in that region, with characteristic cognitive deficits, rather than serve as a systemic risk factor. People with learning disabilities are more likely to develop specific cognitive deficits before progressing to $\mathrm{AD}$.

Finally, adverse childhood events illustrate features of the critical window model. Here the increase in $\mathrm{AD}$ risk depends on timing of an early event. For example, one study suggests that adults who experience maternal death at 11-17 years of age are twice as likely to develop $\mathrm{AD}$ as those without this adverse event.

This approach to early-life risk factors will need refining. It is hard in some cases to assign particular risk factors to a model (for example, this review considers adverse childhood events an example of both the latent and critical window models). Yet consideration of early-life risk factors in AD is important, as an increasing number of studies suggest that many of the diseases of later life are associated with early-life risk factors. As Seifan et al. [2] point out, exposure to adverse childhood events increases the risk of heart disease, stroke and diabetes. Loss of a parent has also been associated with risk of cancer before 40 years of age [5]. Abuse and other adverse events are associated with a variety of medical conditions and poorer physical as well as mental health in adulthood $[6,7]$. We also cannot rule out an intergenerational, in utero factor for some health conditions, as in the Barker hypothesis. Poor brain development related to the pre-pregnancy health of a mother and risk factors affecting the pregnancy (toxic exposures, poor nutrition, lack of social interactions and poor prenatal care) may predispose to a wide variety of health problems, including violence and risk of homicide when these children reach young adulthood.

\section{KARGER 125}

2015 S. Karger AG, Base

0251-5350/15/0454-0255\$39.50/0 
We need more research on early-life risk factors. As Seifan et al. [2] remind us, their review may also be biased in an important way: 'few studies were prospective and survival bias was prominent because early-life factors may cause selective attrition of weaker individuals'. Lifespan cohort studies are required to establish the true risk of early conditions on later de-

\section{References}

1 Sperling RA, Rentz DM, Johnson KA, et al: The A4 study: stopping AD before symptoms begin? Sci Transl Med 2014;6:228fs13.

2 Seifan A, Schelkeb M, Obeng-Aduasare Y, Isaacson R: Early life epidemiology of Alzheimer's disease - a critical review. Neuroepidemiology 2015;45:237-254.

3 Murray AD, McNeil CJ, Salarirad S, Whalley LJ, Staff RT: Early life socioeconomic circumstance and late life brain hyperintensities - a population based cohort study. PLoS One 2014;9:e88969.

4 Noble KG, Houston SM, Brito NH, et al: Family income, parental education and brain structure in children and adolescents. Nat Neurosci 2015; $18: 773-778$ velopment of $\mathrm{AD}$. Until more definitive evidence is available, our efforts are perhaps still best focused on secondary prevention using risk factors with a more definitive place in $\mathrm{AD}$ pathobiology, as in the A4 trial's focus on people with normal cognition who have elevated levels of brain amyloid plaque.

5 Kennedy B, Valdimarsdottir U, Sundstrom K, et al: Loss of a parent and risk of cancer in early life: a nationwide cohort study. Cancer Causes Control 2014;25:499-506.

6 Gilbert LK, Breiding MJ, Merrick MT, et al: Childhood adversity and adult chronic disease: an update from ten states and the district of Columbia, 2010. Am J Prev Med 2015;48:345-349.

7 Wegman HL, Stetler C: A meta-analytic review of the effects of childhood abuse on medical outcomes in adulthood. Psychosom Med 2009;71:805812 . 